\title{
The Pathophysiology of Miscarriage in Women with Polycystic Ovary Syndrome. Review and Proposed Hypothesis of Mechanisms Involved
}

\author{
Paulina A. Essah, Kai I. Cheang, John E. Nestler \\ Departments of Internal Medicine (P.A.E., J.E.N.), Pharmacy (K.I.C.) and Obstetrics and Gynecology (J.E.N.), \\ Medical College of Virginia Campus, Virginia Commonwealth University, Richmond, Virginia 23298
}

\section{INTRODUCTION}

The polycystic ovary syndrome (PCOS) is characterized by chronic anovulation and hyperandrogenism, and is the most common cause of anovulatory infertility in developed countries ${ }^{1,2}$. Affected women suffer not only from infertility, but also from a high rate of early pregnancy loss, defined as miscarriage during the first trimester. The syndrome is associated with a $30-50 \%$ rate of early loss of clinically recognized pregnancies after either spontaneous or assisted concep$\operatorname{tion}^{3-8}$, a rate three-fold higher than that reported for normal women ${ }^{7,8}$. In fact, $36-82 \%$ of women with recurrent pregnancy loss are reported to have PCOS or anatomically polycystic ovaries ${ }^{6}$. In addition to early pregnancy loss, women with PCOS are also at risk of recurrent pregnancy loss, defined as 3 or more consecutive pregnancy losses before 20 weeks gestation. In most cases, no apparent cause of pregnancy loss

Key words: Polycystic ovary syndrome, Insulin resistance, Early pregnancy loss, Recurrent pregnancy loss

Address correspondence and requests for reprints to: John E. Nestler, M.D., Virginia Commonwealth University, MCV Campus, PO Box 980111, Richmond, VA 232998-0111, Tel.: 804-828-9696, Fax: 804-828-8389,

e-mail:nestler@hsc.vcu.edu

Received 30-08-04, Revised 20-09-04, Accepted 25-09-04 can be identified ${ }^{9}$. The specific etiology of pregnancy loss in PCOS remains unknown.

Several factors have been implicated as potential contributors to miscarriage in PCOS. In addition to fetal defects, these include anatomically polycystic ovaries, obesity, placental thrombosis, endometrial defects, and hormonal abnormalities such as excess androgen secretion or insulin resistance. Notably, insulin resistance has been linked to several of the aforementioned contributors to pregnancy loss. In this review, we will first discuss the individual potential contributors to pregnancy loss in PCOS, but in the end, we will provide a unifying theory on the means by which insulin resistance may be central to all of these mechanisms.

\section{FETAL DEFECTS}

While chromosomal abnormalities are believed to frequently be the cause of spontaneous abortion in normal women, little is known about the role of fetal chromosomal defects specifically in women with PCOS. In fact, studies suggest that in PCOS other factors may play a more dominant role in pregnancy loss. In a study ${ }^{10}$ of 41 consecutively examined women with spontaneous abortions occurring within 11 weeks of gestation, fetal chromosomal analyses were conducted on all fetuses. Results demonstrated that women with normal fetal chromosomal karyotypes were more likely than women with abnormal fetal karyotypes to 
have a history of menstrual irregularity $(47.1 \%$ vs. $8.3 \%)$, the presence of polycystic ovaries $(41.2 \%$ vs. $8.3 \%$ ), and elevated basal LH concentrations. These findings suggest that fetal chromosomal abnormalities may not be common in PCOS and, hence, other factors may play a more dominant role in pregnancy loss in this syndrome.

\section{ANATOMICALLY POLYCYSTIC OVARIES}

Polycystic ovaries are a commonly recognized ultrasound abnormality among women with a history of recurrent miscarriages and infertility ${ }^{5,11,12}$. Therefore, the presence of anatomically polycystic ovaries has been postulated to contribute to miscarriage in PCOS, either through ovarian androgen hypersecretion or $\mathrm{LH}$ hypersecretion.

One study of 500 patients with a history of recurrent miscarriages reported a $56 \%$ prevalence rate of polycystic ovaries in this population ${ }^{11}$. A larger cohort study of 2,199 women by the same group of investigators $^{13}$ revealed a $40.7 \%(895 / 2199)$ prevalence rate of anatomically polycystic ovaries among women with recurrent miscarriages. However, no difference in subsequent live birth rates between women with anatomically polycystic ovaries and with normal ovarian morphology was noted, as well as no association of miscarriage with elevated serum LH or testosterone concentrations. Similarly, another study ${ }^{14}$ found a high rate of polycystic ovaries in a population of women with a history of recurrent miscarriages $(36 \%)$ but $82 \%$ of these women proceeded to have subsequent live births, similar to the live birth rate of $81 \%$ encountered in women with normal ovaries.

The conclusion that can be drawn from these studies is that women with polycystic ovaries are overrepresented among women with early miscarriage, implying increased risk, but that among women with an established history of recurrent spontaneous abortion, the presence of polycystic ovaries does not influence the subsequent live birth rate.

\section{OBESITY}

Obesity has also been implicated as a possible risk factor for early pregnancy loss and recurrent miscarriages in PCOS. A retrospective study ${ }^{15}$ divided 712 women who became pregnant after receiving donat- ed oocytes into four groups based on body mass index (BMI). Results showed significant differences in spontaneous abortion rates between obese women $(38.1 \%$, $\left.\mathrm{BMI}=30 \mathrm{~kg} / \mathrm{m}^{2}\right)$ and normal weight $(13.3 \%$, BMI 20$\left.24.9 \mathrm{~kg} / \mathrm{m}^{2}\right)$ or overweight (15.5\%, BMI $25-29.9 \mathrm{~kg} /$ $\mathrm{m}^{2}$ ) women, supporting the concept that obesity is an independent risk factor for spontaneous abortion. Another study ${ }^{16}$ examined the relationship between BMI and the risk of spontaneous abortion in 2,349 women who conceived after assisted reproductive technology treatment. Results revealed that there was a progressively increasing risk of spontaneous abortion as BMI increased. Other studies have corroborated the role of obesity in miscarriage in PCOS ${ }^{17-19}$.

Although obesity has been shown to be a risk factor for pregnancy loss in PCOS, it remains unclear if obesity itself or an obesity-associated comorbidity, such as insulin resistance, is responsible for this phenomenon.

\section{PLACENTAL THROMBOSIS}

Familial thrombophilia (including factor V Leiden mutation, acquired activated protein $\mathrm{C}$ resistance, and antiphospholipid antibody syndrome) and hypofibrinolysis are known to contribute to recurrent pregnancy loss in normal women. Increased activity of plasminogen activator inhibitor-1 (PAI-1), a major inhibitor of fibrinolysis, has been reported to promote recurrent pregnancy loss in normal women. PAI-1 is produced by endothelium and decidualized endometrium, and high PAI-1 activity can result in placental bed thrombosis as well as uterine vascular insufficiency.

Regarding PAI-1 activity in PCOS, Glueck et al. ${ }^{20}$ reported that elevated PAI-1 activity is an independent risk factor for miscarriage in women with PCOS. In their study of 77 pregnancies of 41 women with PCOS, there were 34 miscarriages (44\%) and 42 live births $(55 \%)$. Sixty-seven percent of the women who had miscarriages $(n=12)$ had high PAI-1 activity (in the $95^{\text {th }}$ percentile of normal range) compared with $29 \%$ of women $(n=15)$ with no miscarriages $(\mathrm{p}=$ 0.052). Furthermore, PAI-1 activity and number of pregnancies were statistically determined by stepwise regression to be significant explanatory variables for miscarriage $(p=0.016)$. Compared with normal controls, women with PCOS had a higher prevalence of heterozygous or homozygous polymorphisms of the 
PAI-1 gene locus $(\mathrm{p}=0.028)$.

In a recent study ${ }^{21}$, the same group of investigators reported that PCOS women with a history of recurrent miscarriages, similar to those with single miscarriages discussed above, have high PAI-1 activity. They also reported that the thrombophilic G1691 A Factor V Leiden mutation is associated with recurrent pregnancy loss in women with PCOS. A point mutation in the factor $\mathrm{V}$ Leiden gene can result in activated protein $\mathrm{C}$ resistance, the most common familial thrombophilia. However, these findings have not been confirmed by an independent group.

Not all studies have supported the finding of hypofibrinolysis and thrombophilia in women with recurrent miscarriages. In an observational study ${ }^{22}, 1000$ consecutive women with unexplained recurrent pregnancy loss were compared with a control group of women with no history of recurrent pregnancy loss. Results showed that acquired activated protein $C$ (APC) resistance, but not congenital Factor V Leiden mutation, was associated with miscarriage. In addition to this study, a case control study of 41 women with PCOS and 25 controls conducted in the United Kingdom $^{23}$ reported no difference in the prevalence of APC resistance between the two groups, thereby refuting the theory that women with PCOS are at risk of miscarriage due to thrombosis. Therefore, the theory of placental bed thrombosis as an etiology for miscarriage in PCOS remains speculative and requires further research. A gene defect may not be required since insulin resistance itself has been shown to increase PAI-1 levels.

\section{ENDOMETRIAL DEFECTS}

There has been speculation that endometrial defects may lead to early pregnancy loss in PCOS. Few published studies have specifically investigated this area.

Deficient secretion of endometrial proteins may contribute to miscarriage in PCOS. Glycodelin (previously known as PP14) and IGF binding protein-1 (IGFBP-1) are two proteins secreted by the endometrium that appear to play important roles in endometrial receptivity during implantation and early pregnancy ${ }^{24,25}$. Glycodelin is a glycoprotein produced by secretory and decidualized endometrial glands during the luteal phase that facilitates implantation by inhibiting the immune response of the endometrium to the embryo ${ }^{25-29}$. More specifically, it has been shown to primarily inhibit mixed lymphocyte reaction and natural killer cell activity ${ }^{27,29}$. Both early pregnancy loss and impaired endometrial development are associated with decreased endometrial secretion of glycode$\operatorname{lin}^{30,31}$. IGFBP-1, on the other hand, facilitates adhesion processes at the feto-maternal interface during the periimplantation period ${ }^{32,33}$. Although produced primarily by the liver, IGFBP- 1 is also produced and secreted by the endometrium during pregnancy ${ }^{34}$. Women with PCOS have been shown to have both low serum glycodelin concentrations and serum IGFBP-1 concentrations in pregnancy ${ }^{35}$.

A possible role for insulin has been considered in endometrial defects. In a double blind, placebo-controlled study ${ }^{36}$ of 48 women with PCOS, we demonstrated that the insulin-sensitizer metformin significantly increased follicular and luteal phase serum glycodelin and IGFBP-1 concentrations. In another study $^{37}$, we evaluated 72 women with PCOS and 62 normal controls during the first trimester of pregnancy, and reported that both serum glycodelin and IGFBP-1 concentrations were markedly and significantly lower in women with PCOS. Serum glycodelin was $56 \%$ lower in women with PCOS during gestational weeks 3-5, 23\% lower during weeks 6-8, and similar by weeks $9-11$. Serum IGFBP-1 concentrations were 60-70\% lower in PCOS during weeks 3-5 and 6-8, and $39 \%$ lower during weeks 9-11. This study provided evidence implicating endometrial dysfunction during the peri-implantation period as a possible mechanism for early pregnancy loss in PCOS.

\section{HORMONAL ABNORMALITIES}

\section{Gonadotropins}

Previous studies suggested that women with hypersecretion of luteinizing hormone $(\mathrm{LH})$, a frequent feature of PCOS, are at increased risk for miscarriage after either spontaneous or assisted conception ${ }^{3,4}$. However, it has been more recently demonstrated that in women with elevated circulating LH concentrations and a history of recurrent miscarriage, suppression of endogenous LH release before conception did not improve live birth rates ${ }^{38}$. Other studies have also failed to corroborate elevated $\mathrm{LH}$ as a risk factor for miscarriage in $\mathrm{PCOS}^{39,40}$. 


\section{Androgens}

Elevated androgen levels (testosterone and androstenedione) have been postulated to play a role in miscarriage in PCOS. An earlier study ${ }^{41}$ described an association between elevated androgen concentrations and miscarriage. A more recent study ${ }^{42}$ supported these findings, reporting that plasma concentrations of androgens were significantly higher in women with PCOS who had recurrent miscarriages compared with normal controls. Likewise, women with recurrent miscarriages who did not have PCOS had significantly higher androgen levels than normal controls, suggesting that an elevated androgen profile by itself is involved with recurrent miscarriages. Their results also found a negative correlation between plasma androgen concentrations and glycodelin concentrations from uterine flushings, suggesting that high androgen concentrations may lead to abnormal endometrial development.

Consonant with these reports, a recent in vitro study ${ }^{43}$ demonstrated that androstenedione inhibits endometrial cell growth and secretory activity (specifically glycodelin secretion) in cultured endometrial epithelial cells. The direct effect of androgens on endometrial function was confirmed by demonstrating the presence of androgen receptors in endometrial epithelial cells, and further by showing that the effects of androgens on endometrium were eliminated when cyproterone acetate, an anti-androgen, was added to the cultures.

\section{Insulin Resistance}

Since a frequent feature of PCOS is hyperinsulinemia with insulin resistance, insulin resistance has also been investigated as a causative mechanism for pregnancy loss in PCOS. A recent study suggested that insulin resistance is an independent risk factor for early pregnancy loss in all women ${ }^{17}$, and another that hyperinsulinemia is also an independent risk factor for recurrent pregnancy loss in normal women ${ }^{44}$. This raises the question of whether pregnancy loss in PCOS may be due to insulin resistance. To evaluate the role of insulin resistance in promoting pregnancy loss, two retrospective studies assessed the effects of metformin administration in pregnant women with PCOS.

We conducted a retrospective study ${ }^{45}$ of 65 women with PCOS who received metformin throughout pregnancy and compared their pregnancy outcomes with
31 women with PCOS who did not receive metformin. Results revealed that the rate of early pregnancy loss was significantly reduced in the metformin group compared to the non-intervention group ( $8.8 \%$ in vs. $41.9 \%$, respectively, $\mathrm{p}<0.0001)$. This reduction was even greater in the subset of women with a history of previous miscarriage who received metformin compared to those who did not ( $11.1 \%$ versus $58.3 \%$, respectively, $\mathrm{p}=0.002)$. No evidence of a teratogenic effect of metformin was observed.

The other retrospective study, which utilized historical data for the control, reported similar results ${ }^{46}$. In this study, 72 women with PCOS on metformin had a $17 \%$ rate of early pregnancy loss compared with a past historical rate of $62 \%$ without metformin use in 40 of these 72 women. No miscarriages were reported during the second or third trimesters. Furthermore, metformin therapy during pregnancy did not result in any teratogenic effects or abnormalities of birthweight or early social and motor development. These results must be interpreted with caution since some studies suggest that the likelihood of a subsequent live birth in a woman with PCOS who miscarries may be as high as $82 \%$.

Metformin is classified as a category B medication in pregnancy. In animal studies, metformin is not teratogenic in rats or rabbits at doses two to six times the maximum suggested human daily dose based on body surface area for rats and rabbits, respectively. The studies described as well as another study ${ }^{47}$ suggest that metformin may be safe in pregnant women. However, there remains a need for randomized, prospective, placebo-controlled trials in pregnant women before metformin can be declared safe for routine use in pregnant women with PCOS.

\section{HYPOTHESIS: INSULIN RESISTANCE WITH HYPERINSULINEMIA AS A UNIFYING CAUSE}

Insulin resistance can be associated with all of the mechanisms of early miscarriage that have been previously discussed. Therefore, we propose that insulin resistance with subsequent hyperinsulinemia is a unifying factor in the pathophysiology of miscarriage in PCOS (Figure 1).

Insulin resistance is highly associated with obesity, and since a high proportion of women with PCOS are obese, the hyperinsulinemia resulting from obesi- 


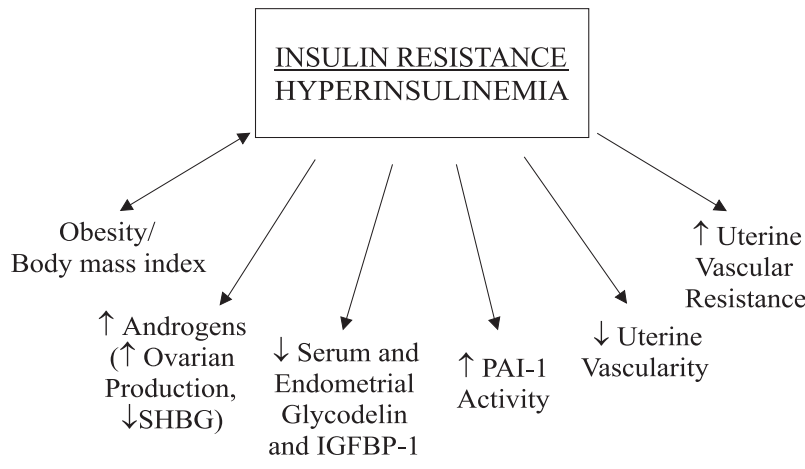

Figure 1. A hypothesis of insulin resistance with hyperinsulinemia as a unifying mechanism for pregnancy loss in PCOS.

ty may directly affect miscarriage rates in PCOS. Support for this theory is derived from a recent study ${ }^{11}$ reporting that obesity is an independent risk factor for early pregnancy loss. The investigators reviewed records of 383 infertile women who conceived after in vitro fertilization or intracytoplasmic sperm injection. After dividing the patients into two weight-based groups (lean, BMI $<25 \mathrm{~kg} / \mathrm{m}^{2}, \mathrm{n}=304$ vs. obese, $\mathrm{BMI}=25 \mathrm{~kg} / \mathrm{m}^{2}, \mathrm{n}=79$ ), they discovered that obese patients, compared with lean women, had a higher rate of miscarriage during the first 6 weeks $(22 \%$ vs. $12 \%, \mathrm{p}=0.03)$ and a lower live birth rate (63\% vs. $75 \%$, $\mathrm{p}=0.04)$. They also reported that the association of obesity and miscarriage is independent of age, history of previous miscarriage, ovarian stimulation method, and the presence of anatomically polycystic ovaries. Other studies on PCOS and infertile women in the general population have supported the finding that obesity is an independent risk factor for early pregnancy $\operatorname{loss}^{18,19}$. It should be noted that it is difficult to distinguish between the effects of obesity and insulin resistance.

In addition to obesity, insulin resistance has been linked to increased expression of PAI-1. PAI-1 activity is known to increase with elevating levels of serum insulin. Conversely, PAI-1 activity has been shown to decrease when insulin concentrations are reduced by metformin ${ }^{20,46,48,49}$. Glueck et al. have postulated that by improving hyperinsulinemia, metformin's reduction in PAI-1 activity reduces spontaneous pregnancy loss in PCOS.

Insulin resistance is known to play a critical role in the ovarian androgen excess characteristics of women with PCOS, and therefore might promote miscarriage by increasing circulating testosterone concentrations. Similarly, increased LH secretion by the pituitary gland may also be a consequence of insulin resistance and the effect of hyperinsulinemia on pituitary function.

Insulin resistance has further been associated with abnormal endometrial development and endometrial defects in women with PCOS. One study ${ }^{36}$ reported that insulin reduction with metformin enhances uterine vascularity and reduces uterine vascular resistance, as demonstrated by a $20 \%$ reduction in vascular resistance in spiral arteries after metformin use $(0.71 \pm$ 0.02 to $0.57 \pm 0.03(\mathrm{p}<0.001)$. This double-blind, placebo-controlled study of 48 women with PCOS also found that insulin reduction with metformin significantly increased follicular and luteal phase serum glycodelin and IGFBP-1 concentrations, two endometrial proteins necessary for implantation and adequate endometrial development that is reduced during the first trimester in women with $\mathrm{PCOS}^{37}$.

In summary, all of the proposed mechanisms for early miscarriage in PCOS can be linked back to insulin resistance and hyperinsulinemia. Hence, insulin resistance may be the initial inciting defect which then results in a cascade of abnormalities, which in combination or individually contributes to the pathogenesis of early miscarriage in PCOS.

\section{CONCLUSION}

In conclusion, while the specific pathophysiology of pregnancy loss in PCOS remains unknown, evidence suggests that factors other than fetal chromosomal abnormalities play a prominent role. We advance the hypothesis that insulin resistance and hyperinsulinemia are essential to the pathophysiology of pregnancy loss in PCOS, acting through a variety of mechanisms. To wit, insulin resistance and hyperinsulinemia have been directly linked to obesity, increased PAI-1 activity, uterovasuclar insufficiency, abnormal gonadotropin secretion, and abnormal endometrial development and function during implantation.

Several areas of investigation are necessary to clarify the etiology of pregnancy loss in PCOS. To determine the individual risk conferred by PCOS on miscarriage, studies comparing miscarriage rates in lean and obese women with PCOS to lean and obese women with normal menses are required. In addition, a large, randomized prospective study would be benefi- 
cial in determining the equality of insulin sensitizing drugs in decreasing miscarriage in PCOS, as well as in identifying any potential safety concerns of these medications during pregnancy. Further studies are needed to understand how insulin might regulate endometrial secretions of glycodelin and IGFBP-1 during early pregnancy.

Nonetheless, the recognition of insulin resistance and subsequent hyperinsulinemia as a central feature of PCOS offers potential insight into a unifying mechanism that may account for the increased risk of early miscarriage in PCOS.

\section{REFERENCES}

1. Asuncion M, Calvo RM, San Millan JL, Sancho J, Avila S, Escobar-Morreale HF, 2000 A prospective study of the prevalence of the polycystic ovary syndrome in unselected Caucasian women from Spain. J Clin Endocrinol Metab 85: 2434-2438.

2. Knochenhauer ES, Key TJ, Kahsar-Miller M, Waggoner W, Boots LR, Azziz R, 1998 Prevalence of the polycystic ovary syndrome in unselected black and white women of the southeastern United States: a prospective study. J Clin Endocrinol Metab 83: 3078-3082.

3. Homburg R, Armar NA, Eshel A, Adams J, Jacobs HS, 1998 Influence of serum luteinising hormone concentrations on ovulation, conception, and early pregnancy loss in polycystic ovary syndrome. BMJ 297: 1024-1026.

4. Regan L, Owen EJ, Jacobs HS, 1990 Hypersecretion of luteinising hormone, infertility, and miscarriage. Lancet 336: 1141-1144.

5. Sagle M, Bishop K, Ridley N, et al, 1988 Recurrent early miscarriage and polycystic ovaries. BMJ 297: 1027-1028.

6. Watson H, Kiddy DS, Hamilton-Fairley D, et al, 1993 Hypersecretion of luteinizing hormone and ovarian steroids in women with recurrent early miscarriage. Hum Reprod 8: 829-833.

7. Gray RH, Wu LY, 2000 Subfertility and risk of spontaneous abortion. Am J Public Health 90: 1452-1454.

8. Regan L, Braude PR, Trembath PL, 1989 Influence of past reproductive performance on risk of spontaneous abortion. BMJ 299: 541-545.

9. Stirrat GM, 1990 Recurrent miscarriage. II: Clinical associations, causes, and management. Lancet 336: 728-733.

10. Hasegawa I, Tanaka K, Sanada H, Imai T, Fujimori R, 1996 Studies on the cytogenetic and endocrinologic background of spontaneous abortion. Fertil Steril 65: 52-54.

11. Clifford K, Rai R, Watson H, Regan L, 1994 An informative protocol for the investigation of recurrent miscarriage: preliminary experience of 500 consecutive cases. Hum Reprod 9: 1328-1332.

12. Kousta E, White DM, Cela E, McCarthy MI, Franks S, 1999 The prevalence of polycystic ovaries in women with infertility. Hum Reprod 14: 2720-2723.
13. Rai R, Backos M, Rushworth F, Regan L, 2000 Polycystic ovaries and recurrent miscarriage - a reappraisal. Hum Reprod 15: 612-615.

14. Liddell HS, Sowden K, Farquhar CM, 1997 Recurrent miscarriage: screening for polycystic ovaries and subsequent pregnancy outcome. Aust N Z J Obstet Gynaecol 37: 402-406.

15. Bellver J, Rossal LP, Bosch E, et al, 2003 Obesity and the risk of spontaneous abortion after oocyte donation. Fertil Steril 79: 1136-1140.

16. Glueck CJ, Streicher P, Wang P, 2002 Treatment of polycystic ovary syndrome with insulin-lowering agents. Expert Opin Pharmacother 3: 1177-1189.

17. Fedorcsak P, Storeng R, Dale PO, Tanbo T, Abyholm T, 2000 Obesity is a risk factor for early pregnancy loss after IVF or ICSI. Acta Obstet Gynecol Scand 79: 43-48.

18. Dale PO, Tanbo T, Haug E, Abyholm T, 1998 The impact of insulin resistance on the outcome of ovulation induction with low-dose follicle stimulating hormone in women with polycystic ovary syndrome. Hum Reprod 70.

19. Hamilton-Fairley D, Kiddy D, Watson H, Paterson C, Franks S, 1992 Association of moderate obesity with a poor pregnancy outcome in women with polycystic ovary syndrome treated with low dose gonadotrophin. Br J Obstet Gynaecol 99: 128-131.

20. Glueck CJ, Wang P, Fontaine RN, Sieve-Smith L, Tracy T, Moore SK, 1999 Plasminogen activator inhibitor activity: an independent risk factor for the high miscarriage rate during pregnancy in women with polycystic ovary syndrome. Metabolism 48: 1589-1595.

21. Glueck CJ, Wang P, Bornovali S, Goldenberg N, Sieve L, 2003 Polycystic ovary syndrome, the G1691A factor V Leiden mutation, and plasminogen activator inhibitor activity: associations with recurrent pregnancy loss. Metabolism 52: 1627-1632.

22. Rai R, Shlebak A, Cohen H, et al, 2001 Factor V Leiden and acquired activated protein $\mathrm{C}$ resistance among 1000 women with recurrent miscarriage. Hum Reprod 16: 961965.

23. Atiomo WU, Condon J, Adekanmi O, Friend J, Wilkin TJ, Prentice AG, 2000 Are women with polycystic ovary syndrome resistant to activated protein C? Fertil Steril 74: 1229-1232.

24. Seppala M, Riittinen L, Julkunen M, et al, 1988 Structural studies, localization in tissue and clinical aspects of human endometrial proteins. J Reprod Fertil 36: 127-141.

25. Julkunen M, Koistinen R, Suikkari AM, Seppala M, Janne OA, 1990 Identification by hybridization histochemistry of human endometrial cells expressing mRNAs encoding a uterine beta-lactoglobulin homologue and insulin-like growth factor-binding protein-1. Mol Endocrinol 4: 700-707.

26. Seppala M, Suikkari AM, Julkunen M, 1988 Human endometrial proteins. Reprod Nutr Dev 28: 1649-1654.

27. Bolton AE, Pockley AG, Clough KJ, et al, 1987 Identification of placental protein 14 as an immunosuppressive factor in human reproduction. Lancet 1: 593-595.

28. Julkunen M, Koistinen R, Sjoberg J, Rutanen EM, Wahl- 
strom T, Seppala M, 1986 Secretory endometrium synthesizes placental protein 14. Endocrinology 118: 17821786.

29. Okamoto N, Uchida A, Takakura K, et al, 1991 Suppression by human placental protein 14 of natural killer cell activity. Am J Reprod Immunol 26: 137-142.

30. Dalton CF, Laird SM, Serle E, et al, 1995 The measurement of CA 125 and placental protein 14 in uterine flushings in women with recurrent miscarriage; relation to endometrial morphology. Hum Reprod 10: 2680-2684.

31. Tulppala M, Julkunen M, Tiitinen A, Stenman UH, Seppala M, 1995 Habitual abortion is accompanied by low serum levels of placental protein 14 in the luteal phase of the fertile cycle. Fertil Steril 63: 792-795.

32. Giudice LC, Mark SP, Irwin JC, 1998 Paracrine actions of insulin-like growth factors and IGF binding protein-1 in non-pregnant human endometrium and at the decidual- trophoblast interface. J Reprod Immunol 39: 133-148.

33. Jones JI, Gockerman A, Busby WHJ, Wright G, Clemmons DR, 1993 Insulin-like growth factor binding protein 1 stimulates cell migration and binds to the alpha 5 beta 1 integrin by means of its Arg-Gly-Asp sequence. Proc Natl Acad Sci USA 90: 10553-10557.

34. Rutanen EM, Koistinen R, Wahlstrom T, Bohn H, Ranta T, Seppala M, 1985 Synthesis of placental protein 12 by human decidua. Endocrinology 116: 1304-1309.

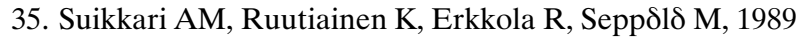
Low levels of low molecular weight insulin-like growth factor- binding protein in patients with polycystic ovarian disease. Hum Reprod 4: 136-139.

36. Jakubowicz DJ, Seppala M, Jakubowicz S, et al, 2001 Insulin reduction with metformin increases luteal phase serum glycodelin and insulin-like growth factor-binding protein 1 concentrations and enhances uterine vascularity and blood flow in the polycystic ovary syndrome. J Clin Endocrinol Metab 86: 1126-1133.

37. Jakubowicz DJ, Essah PA, Seppala M, et al, 2004 Reduced serum glycodelin and insulin-like growth factor-binding protein-1 in women with polycystic ovary syndrome during first trimester of pregnancy. J Clin Endocrinol Metab 89: 833-839.

38. Clifford K, Rai R, Watson H, Franks S, Regan L, 1996 Does suppressing luteinising hormone secretion reduce the miscarriage rate? Results of a randomised controlled trial. BMJ 312: 1508-1511.
39. Thomas A, Okamoto S, O'Shea F, MacLachlan V, Besanko M, Healy D, 1989 Do raised serum luteinizing hormone levels during stimulation for in- vitro fertilization predict outcome? Br J Obstet Gynaecol 96: 1328-1332.

40. Okon MA, Laird SM, Tuckerman EM, Li TC, 1998 Serum androgen levels in women who have recurrent miscarriages and their correlation with markers of endometrial function. Fertil Steril 69: 682-690.

41. Tulppala M, Stenman UH, Cacciatore B, Ylikorkala O, 1993 Polycystic ovaries and levels of gonadotrophins and androgens in recurrent miscarriage: prospective study in 50 women. Br J Obstet Gynaecol 100: 348-352.

42. Okon MA, Laird SM, Tuckerman EM, Li TC, 1998 Serum androgen levels in women who have recurrent miscarriages and their correlation with markers of endometrial function. Fertil Steril 69: 682-690.

43 Tuckerman EM, Okon MA, Li T, Laird SM, 2000 Do androgens have a direct effect on endometrial function? An in vitro study. Fertil Steril 74:771-779.

44 Craig LB, Ke RW, Kutteh WH, 2002 Increased prevalence of insulin resistance in women with a history of recurrent pregnancy loss. Fertil Steril 78: 487-490.

45. Jakubowicz DJ, Iuorno MJ, Jakubowicz S, Roberts KA, Nestler JE, 2002 Effects of metformin on early pregnancy loss in the polycystic ovary syndrome. J Clin Endocrinol Metab 87: 524-529.

46. Glueck CJ, Phillips H, Cameron D, Sieve-Smith L, Wang $\mathrm{P}, 2001$ Continuing metformin throughout pregnancy in women with polycystic ovary syndrome appears to safely reduce first-trimester spontaneous abortion: a pilot study. Fertil Steril 75: 46-52.

47. Coetzee EJ, Jackson WPU, 1984 Oral hypoglycaemics in the first trimester and fetal outcome. S Afr Med J 65: 635-637.

48. Velazquez EM, Mendoza S, Hamer T, Sosa F, Glueck CJ, 1994 Metformin therapy in polycystic ovary syndrome reduces hyperinsulinemia, insulin resistance, hyperandrogenemia, and systolic blood pressure, while facilitating normal menses and pregnancy. Metabolism 43: 647-654.

49. Velazquez EM, Mendoza SG, Wang P, Glueck CJ, 1997 Metformin therapy is associated with a decrease in plasma plasminogen activator inhibitor-1, lipoprotein(a), and immunoreactive insulin levels in patients with the polycystic ovary syndrome. Metabolism 46: 454-457. 